\title{
VALUACIÓN DE LAS PROPIEDADES FÍSICAS Y QUÍMICAS DE RESIDUOS SÓLIDOS ORGÁNICOS A EMPLEARSE EN LA ELABORACIÓN DE PAPEL
}

\author{
Krystle Danitza González Velandia ${ }^{1}$ \\ Dayra Daza Rey² \\ Paola Andrea Caballero Amado ${ }^{3}$ \\ Chadae Martínez González ${ }^{4}$
}

Recibido el 23 de febrero de 2015, aprobado el 29 de octubre de 2015 y actualizado el 25 de mayo de 2016

DOI: 10.17151/luaz.2016.43.21

\section{RESUMEN}

La producción de papel está soportada principalmente en la madera debido a su alto contenido de celulosa. Sin embargo, actualmente se buscan fuentes alternativas que desestimulen el uso de este recurso favoreciendo la protección de los bosques. La celulosa también está presente en las especies vegetales que hacen parte del consumo humano y, por lo tanto, los residuos orgánicos podrían convertirse en materia prima para la fabricación de papel. En Colombia más del $60 \%$ de los residuos que se generan son de carácter orgánico y muy pocos se están aprovechando. Esta investigación se centró en evaluar el contenido de celulosa en diferentes residuos sólidos orgánicos. También, se evaluaron otras propiedades químicas como contenido de hemicelulosa y lignina, y propiedades físicas como densidad, contenido de humedad, entre otros, ya que estas propiedades condicionan los procesos de producción de papel y son importantes para conocer la viabilidad de uso y la estrategia de la extracción de la celulosa. 21 residuos sólidos fueron analizados a través de pruebas de laboratorio basadas en las normas ASTM y TAPPI y se encontró que 9 de estos tienen un alto contenido de celulosa. Tomando en cuenta todos los demás análisis se concluyó que varios residuos tienen alto potencial para ser usados en la producción de papel. El uso de residuos sólidos, además de ser una solución que promueve la protección de los bosques, se puede convertir en una alternativa para el manejo de residuos sólidos en Colombia.

\section{PALABRAS CLAVE}

Celulosa, lignina, papel, residuos orgánicos. 


\title{
EVALUATION OF THE PHYSICAL AND CHEMICAL PROPERTIES OF ORGANIC SOLID WASTE FOR USE IN THE PREPARATION OF PAPER
}

\begin{abstract}
Paper production is supported mainly by the wood due to its high content of cellulose. However, alternative sources that discourage the use of this resource and promoteprotection of forests are currently sough. The cellulose is also present in plants that are part of human consumption and therefore organic waste could become raw material for papermaking. In Colombia more than $60 \%$ of waste that is generated is organic and very few are reused. This research focused on evaluating the content of cellulose in several solid organic waste. Other chemical properties as hemicellulose and lignin content, and physical properties such as density, humidity, among others, were also evaluated because these properties determine the process of paper production and are important to know the feasibility to use and the strategy to extract the cellulose. 21 solid waste were analyzed by laboratory tests based on the ASTM and TAPPI standards and it was found that 9 of these solids have high cellulose content. Taking in account all the other analyses, we concluded that various solid residues have high potential to be used in the production of paper. The use of solid waste could be a solution that promotes the protection of forests and it would become in an alternative for solid waste management in Colombia.
\end{abstract}

\section{KEY WORDS}

Cellulose, lignin, paper, organic waste.

\section{INTRODUCCIÓN}

Dentro de las actividades humanas que están afectando negativamente el ambiente, una de las principales es la asociada al manejo y disposición de residuos sólidos. En el caso de Bogotá, diariamente se generan entre 6000 y 10000 toneladas de residuos que se disponen en el Relleno Sanitario Doña Juana, de los cuales aproximadamente el 60\% son residuos orgánicos (Hernández, 2010; UAESP, 2011). Paralela a esta situación, la industria papelera requiere de grandes cantidades de agua y recursos forestales para la producción de papel, debido a 
que la madera contiene celulosa, fibra con la que se forman las láminas de papel. Se están buscando fuentes alternativas a la madera como la caña de azúcar para disminuir los impactos ambientales, pero la celulosa también se encuentra presente en muchos residuos orgánicos, por lo que esta investigación se centró en determinar las características físicas y químicas de algunos residuos orgánicos para identificar cuáles tienen propiedades para usarse como pulpa de papel. En esta investigación experimental se evaluaron las propiedades relevantes necesarias para la producción de papel como contenidos de humedad, solubilidad en agua caliente y fría, densidades, volumen y las más importantes que determinan su utilidad para la industria como los contenidos de holocelulosa, celulosa y lignina. Esta publicación está estructurada en cinco partes, inicialmente se muestran los antecedentes de usos y evaluaciones de materiales para su uso en la elaboración de papel, los conceptos básicos usados en la industria papelera, la metodología usada y, finalmente, se presentan los resultados obtenidos concluyendo que residuos de los evaluados podrían ser potencialmente usados para la producción de papel.

\section{Antecedentes en el uso de materiales alternativos para producir papel}

La extracción de fibras para hacer papel a partir de fuentes no leñosas no es nueva, a nivel mundial y nacional se han adelantado diferentes estudios sobre fuentes alternativas a la madera como el vástago de plátano, la cabuya, el cáñamo y el algodón, donde se han obtenido resultados satisfactorios utilizando fibras vegetales como pulpa (Peña \& González, 2002; Aguilar, Ramírez \& Malagón, 2007).

Dentro de las investigaciones realizadas, Chiluiza \& Hernández (2009) evaluaron las propiedades físicas y químicas de la Guadua angustifoliainvolucrando dos tipos de parámetros: aspectos apreciables a simple vista y medidos con facilidad, como diámetro, grosor y densidad; y aspectos evaluados en laboratorio como contenido de humedad y la caracterización química, basados en las normas de la Asociación Técnica de la Industria de Pulpa y Papel de Estados Unidos, encontrando en este producto un alto potencial para la industria del papel. En el caso de cítricos por las grandes cantidades de residuos que se generan se han buscado alternativas, un ejemplo en el estudio de Vargas \& Pinoargote (2001) donde se encontró que el papel es una alternativa para aprovechamiento, y aunque en este caso no se puede hablar de la sustitución de pulpa en un 100\%, pues a partir de procesos químicos y mecánicos se concluyó que podía ser viable en un 50\% (relación 50\% fruta: 50\% papel de reciclaje) la sustitución de pulpa. 
La caña de azúcar y el bagazo de maíz son las primeras materias primas utilizadas para la preparación alternativa de pulpa para papel. Se han realizado diferentes estudios para determinar sus contenidos de celulosa, lignina y cenizas para evaluar la pureza de la pulpa, y se ha encontrado que el trabajo con estos residuos requiere de más trabajo para la pulverización y blanqueo lo que es importante en el proceso de industrialización (Prado-Martínez et al., 2012). En el caso de otras fibras fibras no leñosas, Aguilar et al. (2007) concluyeron que cada residuo tiene un comportamiento específico y que, además de considerar el contenido de celulosa, es indispensable considerar otras propiedades. Otros análisis realizados en Colombia se han centrado en la piña y en los residuos de plátano. Hernández (2008) evaluó dos especies de piña (Ananas comosus L. Merr.) y obtuvo como resultado que este papel hecho con la fibra de la corona del fruta puede ser utilizado para muchas funciones por su comportamiento y contenidos de celulosa. Mazzeo, León, Mejía, Guerrero \& Botero (2010) realizaron una caracterización fisicoquímica a los residuos de plátano y para proponer alternativas de aprovechamiento como la obtención de papel a partir del pseudotallo. Todos estos estudios demuestran la importancia de caracterizar física y químicamente los residuos para conocer su potencial y porque de estas características depende el proceso productivo.

\section{Propiedades físicas y químicas del papel}

El papiro fue el origen del uso de papel para facilitar la comunicación y la transmisión de conocimiento, este se componía de láminas del tallo, extraídas, desplegadas y aplanadas. En otras culturas, como las asiáticas, se utilizaba la piel seca de ovejas, cerdos, cabras, entre otras (Bacigalup L., Martino, A. y Soca, A. (2010)).

La estructura básica del papel es un entramado de fibras de celulosa (un polisacárido con 600 a 1000 unidades de sacarosa) unidas mediante enlaces de hidrógeno. Una vez separadas del resto de componentes no celulósicos mediante el proceso de elaboración de la pasta de papel, estas fibras tienen alta resistencia a la tracción, absorben los aditivos empleados para transformar la pasta en papel y cartón, y son flexibles, químicamente estables y blancas. Esos componentes no celulósicos son, en el caso de la madera, principalmente hemicelulosas (con 15 a 90 unidades iguales de sacarosa), ligninas (altamente polimerizadas y complejas, actúan como aglutinante de las fibras), extractos (grasas, ceras, alcoholes, fenoles, 
ácidos aromáticos, aceites esenciales, oleorresinas, esteroles, alcaloides) (Keefe \& Teschke, 1995).

Para la elaboración del papel es determinante conocer las propiedades físicas y químicas de la materia prima a usar, ya que de estas depende directamente la calidad del papel y se establece toda la línea de producción. Dentro de las propiedades físicas que se evalúan comúnmente se encuentran el volumen, tamaño, densidad real y aparente, en el caso de los residuos el grado de madurez, el contenido de humedad y la resistencia, entre otros. Las propiedades químicas son aún más importantes, pues determinan la calidad de las fibras, es decir la resistencia a la tracción, flexibilidad, la estabilidad, la adhesión, el color, absorción de los aditivos empleados para transformar la pasta en papel y cartón (Keefe \& Teschke, 1995; Villaseñor, 2000; Revista Ambientum, 2002). Dentro de las propiedades que se evalúan en la pulpa de papel están la celulosa, la hemicelulosa que rodea los filamentos de la celulosa y ayuda en la formación de microfibrillas, es decir funcionan como material de soporte en la pared celular, la holocelulosa que representa la fracción total de polisacáridos, es decir, celulosa + hemicelulosa, la lignina que es una sustancia natural que hace parte de la pared celular de muchas células vegetales, les da dureza y resistencia pero en el caso del papel se busca eliminarla para facilitar la adhesión de las fibras (Revista Ambientum, 2002). En la Tabla 1 se muestran los resultados de evaluaciones químicas: 
Tabla 1. Materias primas usadas para elaboración de papel

\begin{tabular}{cccc}
\hline Material & Celulosa & Hemicelulosa & Lignina \\
\hline Maderas blandas & $38-46$ & $23-31$ & $22-34$ \\
\hline Maderas duras & $38-49$ & $20-40$ & $16-30$ \\
\hline Paja & $28-42$ & $23-38$ & $12-21$ \\
\hline Bambú & $26-43$ & $25-26$ & $20-32$ \\
\hline Algodón & $80-85$ & n.d. & n.d. \\
\hline Hoja tusa del maíz & $18-40$ & $11,34-31$ & $14-19$ \\
\hline Tallo de clavel & $40-50$ & $25-45$ & $20-25$ \\
\hline Corona piña & $11-45$ & $14-50$ & $10-30$ \\
\hline Tallo rosa & $45-50$ & $20-25$ & $20-25$ \\
\hline Cáscara naranja & 16,2 & 13,8 & 1 \\
\hline Tallo maíz & 50 & 20 & 30 \\
\hline Bagazo plátano & 55,65 & 14 & 11,58
\end{tabular}

Fuente: Keefe \& Teschke (1995), Klass (1998), Sun \& Cheng (2002) y Balat (2011). Adaptado por Quintero (2009); Villalobos \& Sánchez (2010); Juárez, Ramírez, Ramírez, Ramón \& Rodríguez (2011); Sosa, Rivas, Mogollón, Gutiérrez, \& Aguilera (2011); Suesca (2012) y Sánchez, Gutiérrez, Muñoz \& Rivera (2014).

La Tabla 1 presenta los contenidos de celulosa, siendo las maderas las que presentan contenidos más altos, pero indicando que también hay contenidos significativos en otros materiales.

\section{Residuos orgánicos}

Un residuo es cualquier objeto resultante del consumo o uso de un bien en actividades que son susceptibles de aprovechamiento (Decreto 1713 de 2002) (República de Colombia, 2002). Cuando se habla de residuos orgánicos se hace referencia a aquellos residuos que provienen de restos de productos de origen orgánico, y se pueden degradar o desintegrar rápidamente, transformándose en otro tipo de materia orgánica, como los restos de comida, frutas, verduras, huevos, entre otros (UAESP, 2011). Los residuos orgánicos se pueden clasificar según su naturaleza y/o característica física (Jaramillo \& Zapata, 2008) en residuos de alimentos, estiércol, restos vegetales, papel y cartón, cuero, plásticos biodegradables. Al hablar de residuos orgánicos por su origen y contenido es importante identificar las propiedades biológicas (Jaramillo \& Zapata, 2008) como 
constituyentes solubles en agua, tales como azúcares, féculas, aminoácidos y diversos ácidos orgánicos, hemicelulosa, celulosa, grasas, aceites y ceras, lignina, lignocelulosa, proteínas, y los componentes orgánicos pueden ser convertidos biológicamente en gases y sólidos orgánicos relativamente inertes.

\section{METODOLOGÍA}

La investigación inició con la selección de 21 residuos, con la recolección y preparación de las muestras representativas; de cada residuo se recolectaron 1000 g para hacer pruebas físicas y químicas. Se inició con las evaluación de las propiedades físicas, dentro de las propiedades evaluadas están el tamaño calculando tres dimensiones: medida longitudinal (a), diámetro transversal mayor (b) y profundidad (c); se evaluó la forma, es decir, a qué figura geométrica se asemeja, se determinó la densidad real a través de la relación entre masa y volumen real, el volumen real se calculó por medio del método de desplazamiento de agua en una probeta graduada (principio de Arquímedes), se evaluó la estabilidad para indicar si los residuos mantienen una forma o se deshacen fácilmente, y contenido de humedad calculando la relación entre la cantidad de agua que posee cada residuos y su masa seca, se utilizó el método de la estufa calculándolo a través de la fórmula: $\% \mathrm{CH}=$ (Peso del agua contenida en la muestra/peso de la masa seca)*100. Cada una de estas pruebas se repitió seis veces para cada uno de los residuos.

La siguiente etapa de la investigación se centró en evaluar las propiedades químicas que, para el caso de la industria, son determinantes en la elaboración de papel; para la evaluación de estas propiedades se prepararon las muestras, cada muestra fue molida con una picadora y se sometió a un proceso de secado natural por una semana, se trituró y luego se tamizó. Una vez listo el material se utilizó el procedimiento para liberar la muestra de extractivos (componentes solubles en solventes neutros que puedan interferir con algunos análisis químicos posteriores) siguiendo la norma TAPPI 204 (Technical Association of Pulp and Paper Industry Muestra libre de extractivos), una vez listas las muestras se procedió a evaluar la solubilidad en agua caliente a $95^{\circ} \mathrm{C}$ siguiendo el procedimiento de la norma TAPPI T 207 om-93, la solubilidad en agua a temperatura ambiente siguiendo la metodología de la norma TAPPI T 207 om-93. Luego se procedió a estimar el contenido de holocelulosa mediante el método de ASTM D-1104 donde por cada muestra se pesan $2 \mathrm{~g}$ de muestra libre de extractivos, se colocan en un frasco de 
$250 \mathrm{ml}$ y se añade $150 \mathrm{ml}$ de agua, 0,2 $\mathrm{ml}$ de ácido acético y $1 \mathrm{~g}$ de clorito sódico, se coloca en un baño de agua a $70-80^{\circ} \mathrm{C}$ y cada hora durante 5 horas se añaden $0,22 \mathrm{ml}$ e ácido acético muy frío y $1 \mathrm{~g}$ de clorito de sodio; pasadas las cinco horas se coloca en baño de hielo hasta que alcance $10^{\circ} \mathrm{C}$, se filtra el contenido del frasco, se lava con $500 \mathrm{ml}$ de agua fría y se secan los crisoles a $105^{\circ} \mathrm{C}$ hasta peso constante.

Una vez lista la muestra de holocelulosa es posible determinar el contenido de celulosa, en este caso se siguió el procedimiento de la norma ASTM 1695-77, para esta prueba se toman $2 \mathrm{~g}$ de holocelulosa y se agregan $10 \mathrm{ml}$ de hidróxido de sodio al $17,5 \%$ a una temperatura constante de $20^{\circ} \mathrm{C}$ en un baño termorregulador, después de 2 min se agregan $5 \mathrm{ml}$ de solución de hidróxido de sodio en intervalos de 5 min hasta completar $25 \mathrm{ml}$ de hidróxido de sodio incluyendo la cantidad inicial, luego se mezcla a $20^{\circ} \mathrm{C}$ durante $30 \mathrm{~min}$, hasta completar $45 \mathrm{~min}$ y se agregan 33 $\mathrm{ml}$ de agua destilada a $20^{\circ} \mathrm{C}$ y se deja reposar 1 hora antes de filtrar con crisoles lavando con agua destilada. A la celulosa recogida en el crisol se le agregan $15 \mathrm{ml}$ de ácido acético al 10\% y luego se retira el ácido dejando la muestra levemente cubierta por $3 \mathrm{~min}$, luego se seca en el crisol y se determina el peso de la celulosa. Por último se evaluó la lignina insoluble en ácido, se siguió el procedimiento de la norma TAPPI T222 os-74.

Finalmente, con los datos de las propiedades físicas y químicas, se procedió a hacer el respectivo análisis estadístico estimando la media, la desviación estándar para determinar el intervalo de confianza, es decir, un rango de valores en el cual se encuentra el verdadero valor del parámetro, con una probabilidad determinada. Como para este caso se tiene un tamaño de muestra pequeña se procedió a estimar el intervalo de confianza utilizando la distribución de Student t, para un nivel de confianza del 95\% a través de la siguiente educación:

\section{Intervalo de confianza $=X \pm t_{\sqrt{n}}^{8}$}

siendo:

$\mathrm{X}=$ media muestral.

$\mathrm{t}=$ valor de $\mathrm{t}$ que corresponde al intervalo de confianza.

$\mathrm{S}=$ desviación estándar de la muestra.

$\mathrm{n}=$ tamaño de la muestra. 
Con los intervalos de confianza se seleccionaron los datos que estaban en los rangos y con esta información se procedió a identificar qué residuos tienen potencial para utilizarse como pulpa de papel y qué características especiales se deben tener en cuenta para el proceso de producción de papel.

\section{RESULTADOS Y ANÁLISIS}

Los residuos recolectados y seleccionados fueron: capacho de uchuva (Physalis peruviana), tallos de rosa, tallos de clavel, residuos del rábano (Raphanus sativus L.), corona de la piña (Ananas comosus L. Merr.), hoja de la tusa del maíz (Zea mays), pétalos de crisantemo (Chrysanthemum L.), cáscara de naranja (Citrus sinensis), de piña (Ananas comosus L. Merr.), de maracuyá (Passiflora edulis), de lulo (Solanum quitoense), de mango (Mangifera indica), de tomate de árbol (Cyphomandra betacea), de limón (Citrus limon L.), bagazo de maíz (Zea mays), hoja de cebolla larga (Allium fistulosum), tallos de girasol (Helianthus annuus), pétalos de rosas, pellejo de yuca (Manihot esculenta), bagazo de plátano (Musa paradisiacaL.), tallos de clavel (Dianthus caryophyllus), pasto seco (Pennisetum clandestinum). Estos se recolectaron en las plazas de mercado, fruterías y en cultivos aledaños a la ciudad de Bogotá D.C. A cada uno de los residuos se le realizó un análisis físico con 6 repeticiones para caracterizar su estado físico. En la Tabla 2 se muestran los resultados obtenidos. 
Tabla 2. Propiedades físicas de los residuos orgánicos seleccionados

\begin{tabular}{|c|c|c|c|c|c|c|}
\hline \multirow[t]{2}{*}{ RESIDUO } & \multicolumn{3}{|c|}{ DIMENSIONES } & \multirow{2}{*}{$\begin{array}{c}\text { DENSIDAD } \\
\text { APARENTE } \\
\left(g / \mathrm{cm}^{3}\right)\end{array}$} & \multirow{2}{*}{$\begin{array}{c}\text { DENSIDAD } \\
\text { REAL } \\
\left(\mathrm{g} / \mathrm{cm}^{2}\right)\end{array}$} & \multirow{2}{*}{$\begin{array}{c}\text { CONTENIDO } \\
\text { DE } \\
\text { HUMEDAD } \\
(\%)\end{array}$} \\
\hline & $a(\mathrm{~cm})$ & $\mathrm{b}(\mathrm{cm})$ & $c(\mathrm{~cm})$ & & & \\
\hline Capacho de uchuva & $4,1-4,4$ & $4,1-4,33$ & 0,05 & $0,011-0,015$ & $0,59-0,68$ & 27,1 \\
\hline Cáscara de mango & $10,8-13,5$ & $5,6-7,4$ & $0,2 \cdot 0,3^{\circ}$ & $0,93-0,98$ & $1,95-2,2$ & 73 \\
\hline Tallos de rosa & $15,2-36,4$ & $0,5-0,9$ & $0,5-1$ & $0,18-0,19$ & $1,05-1,19$ & 65,3 \\
\hline Tallos de clavel & $25,2 \cdot 35,7$ & $0,8-0,7$ & $0,5-0,9$ & $0,83-0,89$ & $1,71-1,82$ & 84,16 \\
\hline Residuos del rábano & $10,4 \cdot 22$ & $4,4-5,2$ & $0,4 \cdot 0,9$ & $0,05-0,08$ & $2,9-3,02$ & 75,65 \\
\hline Corona de la piña & $6 \cdot 14,3$ & $2,7-5,7$ & $0,4 \cdot 1,3$ & $0,58-0,64$ & $3,44 \cdot 3,69$ & 49,16 \\
\hline Hoja de la tusa del máz & $15-28,3$ & $5,3-6,8$ & $0,1-0,12$ & $0,68-0,075^{\circ}$ & $1,05-1,14^{*}$ & 42,5 \\
\hline Pétalos de crisantemo & $2,5-4,5$ & 0,5 & $0,09-0,1$ & $0,13-0,17^{\circ}$ & $0,61-0,67^{\circ}$ & 66,8 \\
\hline Cáscara de naranja & $13,3-15,2$ & $2,5-5,9$ & $0,8-0,8$ & $0,37-0,37$ & $1,03-1,08$ & 76,74 \\
\hline Cáscara de piña & $6,5-9,1$ & $4,4 \cdot 6,1$ & $0,5-0,9$ & $0,38-0,38$ & $0,7-0,71$ & 84,41 \\
\hline Cáscara de maracuyá & $6,3-9,2$ & 6,4 & $0,8-1,1$ & $0,2 \cdot 0,26$ & $1,09-1,18$ & 86,9 \\
\hline Cáscara de lulo & $12,3-13,5$ & $1,9 \cdot 2,2$ & $0,1-0,8^{\circ}$ & $0,21 \cdot 0,23^{\circ}$ & $0,5-0,53^{\circ}$ & 72,55 \\
\hline Cáscara de t. de árbol & $5,3-7,4$ & $2,0-2,3$ & $0,1-0,9$ & $0,21-0,25$ & $0,52-0,57$ & 69,1 \\
\hline Cáscara de limón & $10,3-14,3$ & $1,4-1,8$ & $0,7=0,9$ & $0,28-0,31^{\circ}$ & $1,28-1,34^{\circ}$ & 63,57 \\
\hline Bagazo de maíz & $45-54,5$ & $12,4-14,1$ & $10,2-12,9$ & $0,38-0,46$ & $2,6-2,82$ & 45 \\
\hline Hoja de cebolla larga & $22-31,6$ & $1,2-1,5$ & $1-1,6$ & $0,04-0,09$ & $2,01-2,08$ & 60,1 \\
\hline Tallos de girasol & $16,8-19,5$ & $1,2-2,3$ & $1,2 \cdot 2,3$ & $0,16-0,18$ & $0,75-0,9$ & 90 \\
\hline Pétalos de rosas & $3,6-5,1$ & $3,1-4,3$ & 0,05 & $0,12-0,16$ & $0,84-0,87$ & 65,25 \\
\hline Pellejo de yuca & $5,5-6,3$ & $21,1-23,2$ & $0,81^{\circ}$ & $0,14-0,17$ & $0,84-0,92$ & 82,09 \\
\hline Vástago plátano & $44-53,6$ & $4,5-5,3$ & $4,5-5,3$ & $0,17-0,21$ & $0,61-0,73$ & 82,5 \\
\hline Pasto seco & $8,5-11,2$ & $0,3-0,5$ & $0,04-0,05$ & $0,06-0,09^{\circ}$ & $1,2-1,31^{*}$ & 25,1 \\
\hline
\end{tabular}

Ciertos residuos se presentan en forma alargada, como el caso de tallos de rosa, de clavel, de girasol, cáscara de limón, de tomate de árbol, de maíz, hoja de cebolla larga, pellejo de yuca, bagazo de plátano, pasto seco. Y otro grupo de residuos, que no presentan una forma específica como la cáscara de mango, corona de la piña, cáscara de piña, de maracuyá, de naranja, residuos de rábano, hoja de la tusa de maíz, tienen tamaños mayores a $5 \mathrm{~cm}$.

Algunos residuos presentan bajos valores de densidad aparente, es decir, poco peso con relación a su volumen, como es el caso del capacho de uchuva, pétalos de crisantemo, cáscara de piña, cáscara de lulo, cáscara de tomate de árbol, tallos de girasol, pétalos de rosas, pellejo de yuca y bagazo de plátano. A diferencia de otros que presentaron altos valores de densidad aparente como cáscara de mango, tallos de rosa, tallos de clavel, residuos del rábano, corona de la piña. 
Al comparar la densidad real con la aparente hay residuos que presentan más diferencias entre estas como capacho de uchuva, residuos del rábano, corona de la piña, hoja de la tusa del maíz y hoja de cebolla larga, es decir que en condiciones normales hay presencia de espacios vacíos entre residuo y residuo, lo que influiría en aspectos como el transporte porque pocas cantidades ocuparían grandes espacios o se requeriría hacer adecuaciones como compactar.

Los contenidos de humedad en su mayoría se encuentran por encima del $60 \%$ debido a que en general se consumen frescos, pero para el caso de la industria del papel altos contenidos de humedad indican alta presencia de agua y menos de fibra que es lo que más interesa en la industria y que, además, influye en el proceso de degradación del residuo y su ablandamiento.

Después de realizar las pruebas físicas se procedió al cálculo de propiedades químicas que para el caso son las más relevantes. Para cada una de las propiedades se realizaron 6 pruebas por cada producto, se identificaron los intervalos de confianza para un nivel de confianza del 95\% y se verificó que los valores medios se encontraran dentro del rango, obteniendo: 
Tabla 3. Propiedades químicas de los residuos orgánicos seleccionados en porcentaje con relación a la materia seca total del residuo en porcentajes

\begin{tabular}{|c|c|c|c|c|c|}
\hline RESIDUO & $\begin{array}{l}\text { PORCENTAJE DE } \\
\text { HOLOCELULOAA }\end{array}$ & $\begin{array}{l}\text { PORCENTANE } \\
\text { DE CELULORA }\end{array}$ & $\begin{array}{l}\text { PORCENTAJE } \\
\text { DE UIVNNA }\end{array}$ & $\begin{array}{l}\text { PORCENTAJE DE } \\
\text { MOLUBLIDAD EN } \\
\text { AGUA FRIA }\end{array}$ & $\begin{array}{l}\text { PORCENTAJE DE } \\
\text { BOLUBIUDAD EN } \\
\text { AGUA CALIENTE }\end{array}$ \\
\hline Pellejo de yuca & 73.77 & $56 \cdot 58^{*}$ & 25,20 & $12-19^{*}$ & $24-27^{*}$ \\
\hline Vástago de plátano & 64.69 & 55.56 & $18-20$ & $14-15$ & $21-26$ \\
\hline Cáscara de pina & $65 \cdot 67$ & $51-55$ & $20-25$ & 29.42 & 55.63 \\
\hline Cáscara de mango & 71.75 & $52-56$ & $30-33$ & $41.45^{*}$ & $60.66^{*}$ \\
\hline Tallos de rosa & $61-64$ & $51-52$ & $25 \cdot 26$ & $12-14$ & $30-35^{*}$ \\
\hline Tallos de maíz & $55 \cdot 62$ & $50-52$ & 32.38 & $8-13$ & $15 \cdot 17$ \\
\hline Cáscara de t. de árbol & 50.59 & 44.45 & $20-25$ & $20-37^{*}$ & $51-56$ \\
\hline Cáscara de lulo & 44.57 & $39-46$ & $26-29$ & $29-56^{*}$ & $55-62$ \\
\hline Pétalos de rosas & 40.59 & 53.47 & $17-22$ & $20-21$ & $38-73$ \\
\hline Tallos de girasol & $52-71$ & $44.46^{i}$ & $15 \cdot 18$ & 7.8 & 30.33 \\
\hline Tallos de clavel & $60-74$ & $39-43$ & $22-30$ & $12-16$ & $25-29$ \\
\hline Cáscara de maracuyá & $45 \cdot 70$ & $32-35$ & 35.43 & $11-15$ & 51.56 \\
\hline Cáscara de naranja & 46.55 & $30-33$ & $8-11$ & $8-13$ & $28-35$ \\
\hline Hoja de cebolla larga & 45.55 & $28-30$ & $17-20$ & $11-19$ & $41-45$ \\
\hline Capacho de uchuva & 40.49 & $26 \cdot 29^{*}$ & $22-26$ & $1-4^{*}$ & $11-25$ \\
\hline Corona de la piña & 44.47 & $25-28$ & $29-30$ & $8-12$ & $52-79$ \\
\hline Pasto seco & 40.44 & $25 \cdot 26$ & $4,8-7$ & $10-19^{*}$ & $45-53$ \\
\hline Residuos del rábano & $25 \cdot 34$ & $20-23$ & $21-26$ & 3.9 & $22-27^{*}$ \\
\hline Pétalos de crisantemo & 25.29 & $19-20^{*}$ & $26 \cdot 35$ & $21-27$ & $42 \cdot 55^{*}$ \\
\hline Cáscara de limón & $17-21$ & $15-17$ & $14-20$ & $6-11$ & $12-15$ \\
\hline Hoja de la tusa del maíz & $25 \cdot 31$ & $7-9$ & $16-23$ & $19-29^{*}$ & $22-28^{*}$ \\
\hline
\end{tabular}

* Casos en los que la media no se ubica en el intervalo de confianza.

Fuente: autores, 2014

Hay residuos que tienen potencial para la producción de papel como es el caso de vástago de plátano, pellejo de yuca, los tallos de rosa, clavel, girasol, de maíz, la cáscara de mango y de tomate de árbol con valores de celulosa por encima del $40 \%$. Es decir que en general los tallos tienen altos contenidos de celulosa, lo que coincide con otras investigaciones como las de Klass (1998), Sun \& Cheng (2002) y Balat (2011) (citados en Suesca, 2012), donde se reportan valores por encima del $40 \%$, es decir que estas fibras están en valores cercanos a maderas blandas y duras.

Algunos residuos presentan valores de celulosa por debajo del $40 \%$ como es el caso del pasto seco, residuos del rábano, pétalos de crisantemo, cáscara de limón, 
de maracuyá, de naranja, hoja de la tusa del maíz, entre otros. Que en el caso de los cítricos coincide con los resultados de Sánchez et al. (2014) en cáscara de naranja con valores por debajo de $30 \%$ en contenidos de celulosa, de igual forma pasa con la hoja de tusa con apenas $8 \%$ en contraste con el estudio de Villalobos \& Sánchez (2010), donde plantean valores ente 18 y 40 y en el caso de pasto con valores por debajo de $30 \%$.

Otro resultado importante a considerar es la lignina porque es necesario reducirla en el proceso de elaboración de papel para que las fibras se puedan adherir y formar la hoja, para eliminarla se necesitan procesos químicos más especializados, en esta caso se puede observar que hay residuos con contenidos de celulosa por encima del $40 \%$ pero también con porcentaje de contenido de lignina por encima del 30\% como los tallos de maíz, de rosa, de clavel, cáscara de mango, de tomate de árbol, de piña, y de lulo que presentan valores que coinciden con autores como Klass (1998), Sun \& Cheng (2002) y Balat (2011) (citados en Suesca, 2012), donde reportan valores de lignina para clavel de $25 \%$ y para cáscara de mango de 30 a $40 \%$, lo que demandaría procesos químicos para retirar la lignina que entrarían a generar mayores impactos sobre el medio ambiente y mayores controles.

El porcentaje de solubilidad en agua caliente y fría influye en el proceso productivo del papel porque hay residuos que se deshacen y facilitan la preparación de la pulpa, como hay residuos en los que hay necesidad de aplicar calor y si este no es suficiente se emplean procesos mecánicos y químicos para deshacer la fibra. Dentro de las fibras con porcentajes de solubilidad en agua fría $\left(18^{\circ} \mathrm{C}\right)$ entre 30 $40 \%$ están los pétalos de crisantemo, cáscara de mango, de lulo, de piña, y de maracuyá asociado a que estos residuos contienen altos porcentajes de pulpa de fruta y por su naturaleza blanda. Estos mismos residuos presentan solubilidad en agua caliente $\left(95^{\circ} \mathrm{C}\right)$ entre $40-60 \%$ sumándose también la corona de la piña, pétalos de crisantemo, pasto seco, corona de la piña, hoja de cebolla larga, cáscara de maracuyá, pétalos de rosas, cáscara de tomate árbol, cáscara de piña. A diferencia residuos como los tallos de clavel, de maíz y de vástago de plátano con contenidos de celulosa por encima del $40 \%$, presentan valores de solubilidad por debajo del $15 \%$, es decir que no se disuelven tan fácilmente lo que combinado con los contenidos de lignina va a determinar un proceso productivo más especializado que con otras fibras, pero con buenos rendimientos con relación a la materia seca. 


\section{CONCLUSIONES}

La caracterización química reveló que los contenidos de celulosa de algunos residuos orgánicos como vástago de plátano, pellejo de yuca, los tallos de rosa, clavel, girasol, de maíz, la cáscara de mango y de tomate de árbol, los convierten en materia prima viable para la producción de papel, remplazando materiales como la madera y podrían convertirse en una solución para los diferentes problemas que se atraviesan con la disposición final de los residuos y para las grandes cantidades generadas en ciudades como la de Bogotá.

No todos los residuos orgánicos son favorables para la obtención de pulpa para papel, de 21 residuos analizados alrededor de 10 tienen contenidos de celulosa por encima del $44 \%$ y 11 no serían potencialmente aprovechables para este proceso, por lo que es importante continuar con más procesos de investigación en búsqueda de su aprovechamiento.

Aunque el contenido de celulosa es un factor determinante para el uso de los residuos orgánicos en la producción de papel, no es la única propiedad que se debe considerar. El proceso productivo depende de otras propiedades como la solubilidad en agua, los contenidos de lignina, la consistencia de los residuos que podrían generar la necesidad de más procesos mecánicos o químicos. Lo importante es desarrollar procesos limpios, al alcance de todas las comunidades, y según las propiedades físicas obtenidas es posible desarrollar estos procesos porque hay residuos que no tienen tan altos contenidos de lignina, como los tallos, cáscaras de frutas, corona de la piña, y para los cuales no es difícil la extracción de la celulosa.

La caracterización física mostró que un aspecto que puede dificultar el aprovechamiento de estos residuos para la elaboración de papel son los altos contenidos de humedad que facilitan el rápido deterioro de los residuos vegetales, por eso las propuestas que se generen deben pensarse en aprovechar el residuo lo más rápido posible, pero en el caso de la producción de papel estos altos contenidos de agua pueden ser un beneficio ya que todo el proceso de producción del papel se desarrolla en presencia de agua. Otra ventaja del uso de los residuos es que estos se muestran en tamaños pequeños lo que facilita los procesos mecánicos de extracción de fibra. 


\section{POTENCIAL CONFLICTO DE INTERESES}

El presente artículo resultado de un proceso de investigación no presenta conflicto de intereses en la realización de la investigación.

\section{FUENTES DE FINANCIACIÓN}

Este proyecto fue financiado por la Dirección de Investigaciones y la Facultad de Ingeniería de la Corporación Universitaria Minuto de Dios.

\section{REFERENCIAS}

- Aguilar, S., Ramírez, J., \& Malagón, O. (2007). Extracción de fibras no leñosas: Cabuya (Furcraea andina Trel.) y Banano (Musa paradisiaca L.) para estandarizar un proceso tecnológico destinado a la elaboración de pulpa y papel. Revista Iberoamericana de Polímeros, 8(2), 89-98.

- Balat, M. (2011). Production of bioethanol from lignocellulosic materials via the biochemical pathway: a review. Energy Convers. Manage., 52(2), 858-875.

- Chiluiza, C. I., \& Hernández, J. P. (2009). Elaboración de papel artesanal de caña guadua (Guadua agustifolia $K$.). (Tesis de grado). Escuela Politécnica Nacional, Quito, Ecuador. Recuperado de http://bibdigital.epn.edu.ec/bitstream/15000/1901/1/CD-2457.pdf

- Hernández, M. (2008). Elaboración y caracterización del papel artesanal de la corona del fruto de dos variedades de piña Ananas comosus (L.) Merr. (Tesis de grado). Universidad Autónoma de Chapingo, Texcoco, México. Recuperado

de http://portal.chapingo.mx/dicifo/tesislic/2008/hernandez_ortega_maricela_2008. pdf

- Hernández, R. (2010, 9 de agosto). Las basuras y el reciclaje en Bogotá. La Silla Vacía. Recuperado de 
http://www.lasillavacia.com/elblogueo/blogverde/17918/las-basuras-y-elreciclaje-en-bogota

- Jaramillo, G., \& Zapata, L. M. (2008). Aprovechamiento de los residuos sólidos orgánicos en Colombia. (Tesis de especialista). Universidad de Antioquia, Medellín, Colombia. Recuperado dehttp://tesis.udea.edu.co/dspace/bitstream/10495/45/1/AprovechamientoRSOUenColombia. pdf

- Juárez, J. M., Ramírez, E. de J.; Ramírez, E., Ramón, L. G., \& Rodríguez, J. (2011). Aplicación y comparación de pretratamientos totalmente libres de cloro en residuos de piña (Ananas comosus) y zapote mamey (Pouteria sapota) para la obtención de carboximetilcelulosa. Revista Venezolana de Ciencia y Tecnología de Alimentos, 2(1), 108-126. Recuperado de http://www.researchgate.net/publication/246044464

_Aplicacin_y_comparacin_de_pretratamientos_totalmente _libres_de_cloro_en_residuos_de_pia_(Ananas_comosus) _y_zapote_mamey_(Pouteria_sapota)_para_la_obtencin_ de_carboximetilcelulosa

- $\quad$ Keefe, A., \& Teschke, K. (1995). Industria del papel y de la pasta de papel. Sectores basados en recursos biológicos. Recuperado de http://www.insht.es/InshtWeb/Contenidos/Documentacion/ TextosOnline/EnciclopediaOIT/tomo3/72.pdf

- Klass, D. L. (1998). Biomass for renewable energy, fuels and chemicals. San Diego, CA: Ed. Academic Press.

- Mazzeo, M., León, L.; Mejía, L. F., Guerrero, L. E., \& Botero, J. D. (2010). Aprovechamiento industrial de residuos de cosecha y poscosecha de plátano en el departamento de Caldas.Revista Educación en Ingeniería, 9, 128-139.

- Peña Giraldo J. y González Peña R., (2012). Estudio de prefactibilidad para la producción de pulpa para papel aprovechando los desechos del cultivo del plátano en la región del viejo caldas. Universidad de Antioquia, 136. http://www.bdigital.unal.edu.co/1954/1/joseabadpenagiraldo.2002.pdf

- Prado-Martínez, M., Anzaldo-Hernández, J., Becerra-Aguilar, B., PalaciosJuárez, H., Vargas-Radillo, J. de J., \& Rentería-Urquiza, M. (2012). 
Caracterización de hojas de mazorca de maíz y de bagazo de caña para la elaboración de una pulpa celulósica mixta.Madera y Bosques, 18(3), 3751.http://redined.mecd.gob.es/xmlui/bitstream/handle/11162/107/00120093001110.pdf?sequ ence $=1$

- Quintero, R. (2009, 19 de junio). Biocombustibles lignocelulósicos: Estado del arte.Segundo Seminario de Divulgación. Ventajas y Desventajas de las Energías Alternativas: El Caso de los Biocombustibles. México: INE, SEMARNAT.

- Bacigalup L., Martino, A. y Soca, A. (2010) El papel Fundamentos de la enseñanza de este soporte en el área del diseño y la comunicación visual. II Congreso Iberoamericano de Investigación Artística y Proyectual y $\mathrm{V}$ Jornada de Investigación en Disciplinas Artísticas y Proyectuales (La Plata, 2010). ISSN: 1850-6011. La Plata, 2010. Recuperado de: http://sedici.unlp.edu.ar/handle/10915/39383

- República de Colombia. (2002). Decreto 1713 de 2002. Por el cual se reglamenta la Ley 142 de 1994, la Ley 632 de 2000 y la Ley 689 de 2001, en relación con la prestación del servicio público de aseo, y el Decreto Ley 2811 de 1974 y la Ley 99 de 1993 en relación con la Gestión Integral de Residuos Sólidos. http://www.alcaldiabogota.gov.co/sisjur/normas/Norma1.jsp?i=5542

- Revista Ambientum. (2002). Plan de Residuos Industriales 2002-2010 de Castilla León. Recuperado de http://www.ambientum.com/revista/2003_05/RSDINDUSTRIALES.htm

- Sánchez, A. M., Gutiérrez, A. I., Muñoz, J. A., \& Rivera, C. A. (2014, 25 de marzo). Producción de bioetanol a partir de subproductos agroindustriales lignocelulósicos. revista TUMBAGA. Vol. 2, núm. 8 (2013) Recuperado de: http://revistas.ut.edu.co/index.php/tumbaga/article/viewFile/58/58

- Sosa, A., Rivas, J., Mogollón, G., Gutiérrez, I., \& Aguilera, A. (2011). Evaluación papelera del pseudotallo de plátano (Musa paradisiaca) en formulaciones con Hevea brasiliensis, Eucalyptus urophylla y Pinus caribaea var. hondurensis. Revista Forestal Venezolana, 55(1), 9-16. 
- Suesca, A. (2012). Producción de enzimas celulolíticas a partir de cultivos de Trichoderma sp. con biomasa lignocelulósica. (Tesis de magíster). Universidad Nacional de Colombia, Facultad de Ingeniería, Departamento de Ingeniería Química y Ambiental, Bogotá, Colombia. Recuperado de http://www.bdigital.unal.edu.co/7843/1/300054.2012.pdf

- Sun, Y., \& Cheng, J. (2002). Hydrolysis of lignocellulosic materials for ethanol production: a review. Bioresource Technology, 83, 1-11.

- UAESP -Unidad Administrativa Especial de Servicios Públicos-. (2011). Caracterización de los residuos sólidos residenciales generados en la ciudad de Bogotá D.C. 2011. Alcaldía Mayor de Bogotá. Recuperado dehttp://www.uaesp.gov.co/uaesp_jo/attachments/Caracterizaci\%C3\%B3n/RESIDENCIALES \%2002-29-2012(!).pdf

- Vargas, K., \& Pinoargote, G. (2001). Aprovechamiento de subproductos de la industrialización de cítricos. Costa Rica: Universidad EARTH.

- Villalobos, L., \& Sánchez, J., (2010). Evaluación agronómica y nutricional del pasto Ryegrass perenne tetraploide (Lolium perenne) producido en lecherías de las zonas altas de Costa Rica. II. Valor nutricional. Agron. Costarricense, 34(1), 43-52.

- Villaseñor, J. y Rutiaga, J. (2000). La madera de Casuarina equisetifolia L., química e índices de calidad de pulpa. Madera y Bosques, vol. 6, núm. 1, 2000, pp. 29-40 Instituto de Ecología, A.C. Xalapa, México. Recuperado de: http://www.redalyc.org/pdf/617/61760103.pdf

1. Magíster en Gestión Ambiental y Desarrollo Sostenible. Docente de la Facultad de Ingeniería, Corporación Universitaria Minuto de Dios. Bogotá, Colombia.kgonzalez@uniminuto.edu. ORCID: 0000-0002-6982-2569

2. Estudiante de Ingeniería Agroecológica. Corporación Universitaria Minuto de Dios. Bogotá, Colombia. dayradaza14@hotmail.com. ORCID: 0000-0002-2710-231 3. Estudiante de Ingeniería Agroecológica. Corporación Universitaria Minuto de Dios. Bogotá, Colombia. shadae_mart20@hotmail.com. ORCID: 0000-0002-7504082 
4. Estudiante de Ingeniería Agroecológica. Corporación Universitaria Minuto de Dios. Bogotá, Colombia. paolacaballero7@gmail.com. ORCID: 0000-0002-92434206

Para citar este artículo: González Velandia, K. D., Daza Rey, D., Caballero Amado, P. A., \& Martínez González, C. (2016). Evaluación de las propiedades físicas y químicas de residuos sólidos orgánicos a emplearse en la elaboración de papel. Revista Luna Azul, 43, 499-517. Recuperado de http://200.21.104.25/lunazul/index.php?option=com_content\&view=article\&id=21 0

Esta obra está bajo una Licencia de Creative Commons Reconocimiento CC BY (c) (i) 